\title{
Serum trans-fatty acids level are positively associated with lower food security among american adults
}

\author{
Mohsen Mazidi ${ }^{1,2}$ and Hassan Vatanparast ${ }^{3}$
}

\begin{abstract}
Objectives: In the current study we aimed to assess whether the food security is associated with serum trans-fatty acids (TFAs) and dietary fat.

Methods: Analyses were restricted to participants (from the US National Health and Nutrition Examination Survey) with data available on serum and diet TFAs and food security status from 2009 to 2010 . All statistical analyses (analysis of covariance and linear regression) accounted for the survey design and sample weights.

Results: We included 3876 participants, overall (48.6\%) participants were men, and (51.4\%) were women, generally (69.0\%) had high food security. Subjects with higher food security had a higher level of education as well $(p<0.001)$. Age-adjusted, sex-adjusted, race-adjusted, education-adjusted mean of trans 9-octadecenoic acid and trans-9, trans12 -octadecadienoic acid were higher in plasma of participants with lower food security (all $p<0.001$ ), moreover in same model there was a significant positive association between plasma level of trans-11-octadecenoic acid, trans-9octadecenoic acid and trans-9, trans-12-octadecadienoic acid and score of food security. Further, age, sex, race, education, and energy intake adjusted mean of dietary fatty acids show that total polyunsaturated fatty acids are higher in subjects with higher food security $(p=0.026)$ while, cholesterol consumption is higher in subjects with lower food security $(p=0.039)$.
\end{abstract}

Conclusions: Our findings provide more evidence on the association between food insecurity and the higher level of TFAs in serum and different type of fat in the diet.

\section{Introduction}

Trans-fatty acids (TFAs) are unsaturated fatty acids with at least one unsaturated, non-conjugated double bond in the trans (rather than the typical cis) configuration. Because humans do not synthesize TFAs, levels of these fatty acid isomers in serum depend on dietary intake. TFAs occur naturally in fat from ruminant animal

\footnotetext{
Correspondence: Hassan Vatanparast (vatan.h@usask.ca)

${ }^{1}$ Key State Laboratory of Molecular Developmental Biology, Institute of

Genetics and Developmental Biology, Chinese Academy of Sciences, Beijing 100101, China

${ }^{2}$ Institute of Genetics and Developmental Biology, International College, The University of Chinese Academy of Science, Beijing 100101, China

Full list of author information is available at the end of the article
}

meat, milk, and dairy fat and artificially in industrially hardened vegetable oils ${ }^{1}$. Increased dietary intake of TFAs is linked with the incident of cardiovascular disease (CVD), diabetes mellitus and atherosclerosis ${ }^{2-5}$. It has been suggested that plasma TFAs concentrations may reflect body fatty acid composition, quality of dietary fat and the type of fat consumed over a long period ${ }^{6,7}$. A recent observational study ${ }^{8}$ and a short-term randomized controlled trial ${ }^{9}$ have indicated that TFA intake increases systemic inflammation in generally healthy individuals. In addition, it has been reported that systemic inflammation is counted as an independent risk factor for the risk of future $\mathrm{CVD}^{10}$, taking together the information can provide us a novel potential insight into the crucial role of the 
TFAs which may affect the cardiovascular health in generally healthy subjects.

As defined by Food and Agriculture Organization of the United Nations "Food security is a situation that exists when all people, at all times, have physical, social and economic access to sufficient, safe and nutritious food that meets their dietary needs and food preferences for an active and healthy life"11. The data from the 2013 Current US Population Survey showed that, nationally, $15.8 \%$ of people were food insecure ${ }^{12}$. Food security exhibits itself in a limited capability to buy healthy foods (e.g., vegetables, fruits, and animal products) because of their greater price compared to fast foods and junk foods high in fat and added sugar (cheaper) ${ }^{13,14}$. Latin-American studies have found an association between food security and poorer dietary quality among adults, particularly low intake of fruits, vegetables, meat, and dairy products ${ }^{14-16}$. Currently, food security counted as one of the main social determinants of health ${ }^{17}$.

Food insecurity, as a social determinant of health, is another marker of socioeconomic distress that has been associated with CVD risk factors and other outcomes in recent years ${ }^{18-21}$. However, the underlying mechanism is not yet known. As there is a strong association between food consumption patterns and some non-communicable diseases such as metabolic syndrome (MetS) ${ }^{22-24}$ and $\mathrm{CVD}^{25-27}$, the inexpensive, low quality of food commonly used in food insecure households could be a link between food security with Mets and CVDs.

Given to importance of the role of TFAs via inflammation in the pathology of CVD and atherosclerosis, we hypothesize that individuals with higher food security should possibly have a lower level of TFAs in their serum reflecting their consumption pattern of fatty acids consumption. Our results could shed light to better understand the pathways that explain the reported association between food security and CVD, atherosclerosis, and $\mathrm{MetS}^{28-30}$. A better understanding of the association between food security and serum TFAs can potentially inform policy makers and dietitians to direct interventions facilitate availability and accessibility of high-quality food fro at risk population. Therefore, we aimed to examine the association between food security status with serum TFAs and also fatty acids intake by using a nationally representative sample of American adults.

\section{Materials and methods}

This is a cross-sectional study using the publically available National Health and Nutrition Examination Survey (NHANES) data set. NHANES is an ongoing survey of a nationally representative sample of the US population with details available elsewhere ${ }^{31-35}$. All participants were requested to provide informed consent, and the National Centre for Health Statistics Research Ethics
Review Board approved the protocol. Since the data is publically available, the study was exempted from obtaining additional local institutional ethics review board approval. We combined four 2-year NHANES survey cycles from 2009 to 2010. We Included subject with higher or equal to 18 years of age. Details on NHANES Laboratory/Medical technologists procedures, anthropometry, laboratory procedures, collection, storage, calibration and quality control of blood samples are described elsewhere $^{35-37}$. (http://www.cdc.gov/NCHS/data/nhanes/ nhanes_09_10/CRP_F_met.pdf. [accessed 19.08.13]).

\section{Plasma TFAs}

A blood sample was drawn from the participant's antecubital vein by a trained phlebotomist according to a standardized protocol ${ }^{33}$, 38 . Plasma TFAs were measured as part of the NHANES protocol and assessed the total (free and esterified) content of selected TFAs in plasma and provides results in concentration units ${ }^{39}, 40$. In this method, the fatty acids in plasma are converted into free fatty acids by subsequent acidic and alkaline hydrolysis. Free fatty acids are extracted using liquid-liquid extraction and derivatized with pentafluorobenzyl bromide ${ }^{39,41}$. The derivatized fatty acids are separated by capillary gas chromatography and detected by mass spectrometry using negative chemical ionization. The fatty acids are identified based on their chromatographic retention time and on the specific mass to charge ratio of the ion formed in the ion source. Retention times are then compared against those obtained with known standards ${ }^{42}$. Quantitation is performed with a standard solution using stable isotope-labeled fatty acids as internal standards. To calculate TFAs as a percent of total fatty acids, 29 fatty acids were determined by this measurement procedure. These fatty acids comprise $95 \%$ of all fatty acids present in plasma $^{43}$. This method determines the following four TFAs: trans-9-hexadecenoic acid (palmitelaidic acid, C16:1n-7t), trans-9-octadecenoic acid (elaidic acid, C18:1n-9t), trans-11-octadecenoic acid (vaccenic acid, C18:1n-7t-), trans-9, trans-12-octadecadienoic acid (linolelaidic acid, C18:2n-6t, 9t) ${ }^{39}$. More detailed information on the NHANES protocol can be found in NHANES manual. (https://www.cdc.gov/Nchs/Data/ Nhanes/Nhanes_99_00/TFA_A_trans_fatty_acids_met. pdf.)

\section{Food security}

More than $99 \%$ of the eligible sample participated in the Food Security Survey Module, which is a well-validated questionnaire developed by the US Department of Agriculture (USDA) to measure household food security over the prior 12 months ${ }^{44}$. Using validated cut points, we categorized the subjects as (a) adult full food security: no affirmative response in any of these items, (b) Adult 


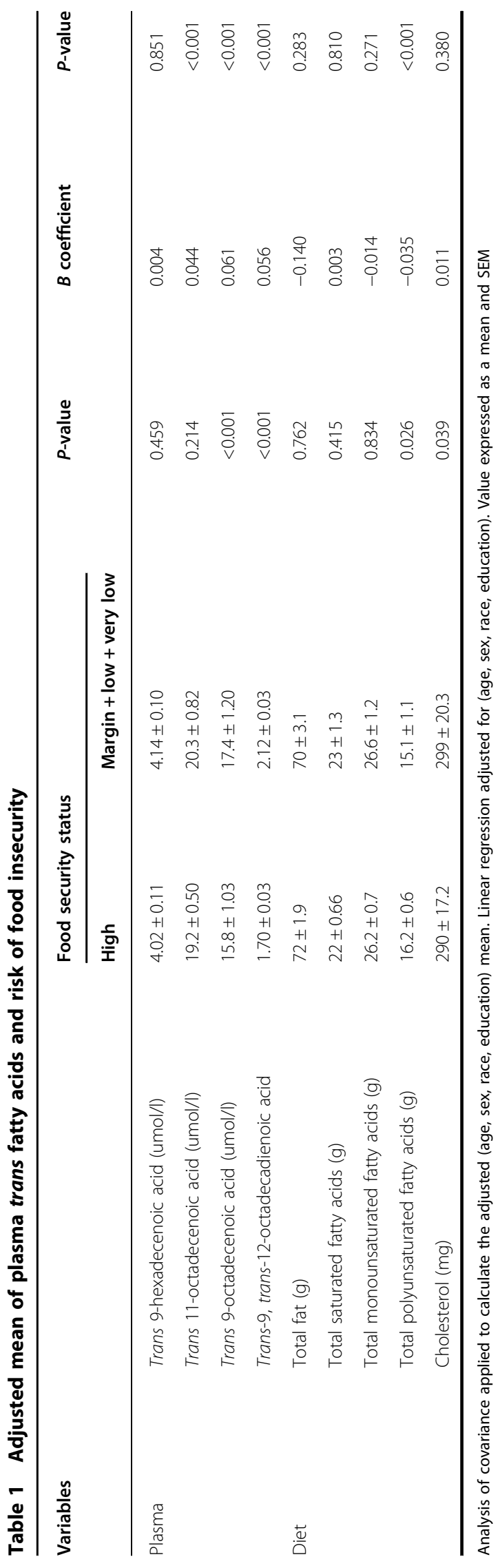

marginal food security: 12 affirmative responses. (c) Adult low food security: 3-5 affirmative responses, (d) Adult very low food security: $6-10$ affirmative responses ${ }^{44}$.

\section{Statistical analysis}

We used the guidelines set by the Centers for Disease Control and Prevention for analysis of the NHANES data set, to analyze the data accounting for the masked variance and using their suggested weighting methodology $y^{35}$, ${ }^{45}$. Age-, education-adjusted, sex-adjusted and the raceadjusted mean of plasma TFAs were compared across categories of food security (high food security vs. food insecurity) using analysis of covariance (ANCOVA). Adjusted (age, sex, education, and race) linear regression applied to examine the association between food security score with plasma TFAs levels. All tests were two-sided, and alpha was set at $p<0.05$ presenting the level of significance unless otherwise stated. We have applied complex sample strategies, to deal with sample weights, unequal probabilities of selection, nonresponse bias, and oversampling. SPSS software (version 18, Chicago, IL, USA) was used for statistical analysis.

\section{Results}

A total of 10,537 participants took part in NHANES across the years under consideration, of which 3876 were eligible for inclusion in the current analyses. From the eligible sample, (69.0\%) had high food security. Overall (48.6\%) participants were men, and (51.4\%) were women. Compared to those with high food security, participants with low food security comprised more of the minority ethnic groups including Mexican-Americans (27.4 vs. 14.9\%), other Hispanic (14.3 vs. 8.2\%), non-Hispanic Black (23.0 vs. $16.2 \%)$, and fewer non-Hispanic White (30.4 vs. $54.7 \%) ; p<0.001$ for differences in the distribution of ethnicity by status for food security. The mean age was 48.1 years overall; and the age was higher in participants with high food security than in those without (50.5 vs. 42.7 years, $p<0.001$ ). Participants with high food security were mostly in group with higher education level, "less than high school" (high food security $=8.6 \%$ vs. low food security $=20.9 \%$ ), "completed high school" (high food security $=35.3 \%$ vs. low food security $=47.7 \%$ ) and "more than high school" (high food security $=55.9 \%$ vs. low food security $=31.2 \%) ; p<0.001$ for differences in the distribution of education level by status for food security.

Using ANCOVA, we computed adjusted (age, sex, race, education) mean of the plasma TFAs for the level of the food security. The level of trans-9-octadecenoic acid and trans-9, trans-12-octadecadienoic acid was higher in plasma of participants with lower food security (all $p<$ 0.001, Table 1).

There was a significant positive association between plasma level of trans-11-octadecenoic acid, trans-9- 
octadecenoic acid and trans-9, trans-12-octadecadienoic acid and score of food security, that is, subjects with a higher score, lower food security, have a higher level of TFAs in plasma. Further, age, sex, race, education, and energy intake adjusted mean of dietary fatty acids show that total polyunsaturated fatty acids (PUFA) are higher in subjects with higher food security $(p=0.026$, Table 1$)$ while cholesterol consumption is higher in subjects with lower food security $(p=0.039$, Table 1$)$. There was also a significant association between dietary PUFA and the level of food security $(\beta=-0.035, p<0.001$, Table 1$)$.

\section{Discussion}

The present study evaluates the association between plasma TFAs level with food security in US population. Significant and inverse association between plasma level of trans-11-octadecenoic acid, trans-9-octadecenoic acid, and trans-9, trans-12-octadecadienoic acid and food security is the main finding of this study. In addition, the consumption of PUFA was higher in participants with higher food security status.

In line with our findings, an investigation in US population reported that food-insecure adults have a higher consumption of high-dairy products. Also, food insecurity was significantly associated with lower Healthy Eating Index, consequently leading to increasing chronic disease risk $^{46}$. In another cross-sectional study from the California Health Interview Survey, food insecurity was significantly associated with lower consumption of fruits and vegetable $^{47}$. In addition, food-insecure individuals had a high total fat intake ${ }^{48}$.

The tool used in NHANES, Household Food Security Survey Module, is measuring income-related household food security. This reflects the fact that disadvantaged groups of the population who have lower socio-economic status are more at risk of food insecurity. A study among Vietnamese reported that food insecurity was inversely associated with total household income and fruit intakes ${ }^{49}$. The cost of high-quality food such as fresh fruit and vegetable is the main factor limiting in at-risk population leading to the consumption of more fast food and junk foods high in trans-fat and saturated fat and sugar and low in micronutrients intake leading to a higher prevalence of inadequacy in nutrient intake and iron deficiency anemia ${ }^{50}$. Conversely, foods with high calorie which mostly consumed by those with low food security might contribute to obesity, and raise the likelihood of the NCDs, such as T2D and CVD ${ }^{50}$. Recent reports from NHANES 2003-2010 stated that foodinsecure adolescents consume more fast foods in comparison with the food-secure population ${ }^{51}$. It has been reported that the high intake of the foods that are loaded with a great amount of the saturated fats may lead to deterioration of the blood lipid profile. Moreover, in study conducted in adolescents, a higher odds of the having elevated Total cholesterol was reported in participants with diets that was highly loaded with sugars including sucrose and fructose ${ }^{52}$.

While the association between food insecurity and chronic conditions has been reported in epidemiologic studies $^{18-20}$, the pathophysiologic pathway is not clear. Our finding that food insecure adults have a higher serum level of TFAs compared to food secure individuals may partly explain such association due to the proinflammatory role of TFAs. Therefore, public awareness, initiatives, and policies that limit the TFAs level in diet can be a reasonable approach for improving diet quality, in general, and among food insecure households in particular, and preventing its related diseases.

\section{Acknowledgements}

MM was supported by a TWAS studentship of the Chinese Academy of Sciences. Financial Disclosure: None.

\section{Author details}

'Key State Laboratory of Molecular Developmental Biology, Institute of Genetics and Developmental Biology, Chinese Academy of Sciences, Beijing 100101, China. ${ }^{2}$ Institute of Genetics and Developmental Biology, International College, The University of Chinese Academy of Science, Beijing 100101, China. ${ }^{3}$ College of Pharmacy and Nutrition/School of Public Health, University of Saskatchewan, Saskatchewan, Canada

\section{Competing interests}

The authors declare that they have no competing financial interests.

\section{Publisher's note}

Springer Nature remains neutral with regard to jurisdictional claims in published maps and institutional affiliations.

Received: 8 May 2017 Accepted: 20 September 2017

Published online: 07 March 2018

\section{References}

1. Sommerfeld, M. Trans unsaturated fatty acids in natural products and processed foods. Prog. Lipid Res. 22, 221-33 (1983).

2. Stender, S. \& Dyerberg, J. Influence of trans fatty acids on health. Ann. Nutr. Metab. 48, 61-6 (2004).

3. Zhao, X. et al. Trans-fatty acids aggravate obesity, insulin resistance and hepatic steatosis in C57BL/6 mice, possibly by suppressing the IRS1 dependent pathway. Molecules 21, 705 (2016).

4. Mozaffarian, D., Aro, A. \& Willett, W. C. Health effects of trans-fatty acids: experimental and observational evidence. Eur. J. Clin. Nutr. 63, S5-21 (2009).

5. Barnard, N. D., Bunner, A. E. \& Agarwal, U. Saturated and trans fats and dementia: a systematic review. Neurobiol. Aging 35, S65-73 (2014).

6. Lee, H. Y., Woo, J., Chen, Z. Y., Leung, S. F. \& Peng, X. H. Serum fatty acid, lipid profile and dietary intake of Hong Kong Chinese omnivores and vegetarians. Eur. J. Clin. Nutr. 54, 768-73 (2000).

7. Sahebkar, A. et al. Lipid-modifying effects of nutraceuticals: an evidence-based approach. Nutrition 32, 1179-92 (2016).

8. Mozaffarian, D. et al. Dietary intake of trans fatty acids and systemic inflammation in women. Am. J. Clin. Nutr. 79, 606-12 (2004).

9. Baer, D. J., Judd, J. T., Clevidence, B. A. \& Tracy, R. P. Dietary fatty acids affect plasma markers of inflammation in healthy men fed controlled diets: a randomized crossover study. Am. J. Clin. Nutr. 79, 969-73 (2004).

10. Libby, P., Ridker, P. M. \& Maseri, A. Inflammation and atherosclerosis. Circulation 105, 1135-43 (2002) 
11. FAO. The State of Food Insecurity in the World 2001, Food and Agricultural Organization (Rome, 2002).

12. Coleman-Jensen, A., Gregory, C., Singh, A. Household food security in the United States in 2013. Food and Agricultural Organization, Roma, USDA-ERS Economic Research Report (2014).

13. Monsivais, P., Aggarwal, A. \& Drewnowski, A. Are socio-economic disparities in diet quality explained by diet cost? J. Epidemiol. Community Health $\mathbf{6 6}, 530-5$ (2012).

14. Rodriguez, L. A., Mundo-Rosas, V., Mendez-Gomez-Humaran, I., Perez-Escamilla, R., Shamah-Levy, T. Dietary quality and household food insecurity among Mexican children and adolescents. Mater. Child Nutr. 13 https:/doi.org/ 10.1111/mcn.12372. [Epub 2016 Nov 14]. (2016).

15. Kirkpatrick, S. I. et al. Household food insecurity is a stronger marker of adequacy of nutrient intakes among Canadian compared to American youth and adults. J. Nutr. 145, 1596-603 (2015).

16. Mark, S., Lambert, M., O'Loughlin, J. \& Gray-Donald, K. Household income, food insecurity and nutrition in Canadian youth. Canadian journal of public health = Revue canadienne de sante publique 103, 94-9 (2012).

17. Mikkonen, J. R. D. Social Determinants of Health: The Canadian Facts. (York University school of health policy and management, Toronto, 2010.

18. Ford, E. S. Peer reviewed: Food security and cardiovascular disease risk among adults in the United States: findings from the National Health and Nutrition Examination Survey, 2003-2008. Prev. Chronic Dis. 10, E202 (2013).

19. Liu, K. et al. Healthy lifestyle through young adulthood and the presence of low cardiovascular disease risk profile in middle age the coronary artery risk development in (young) adults (cardia) study. Circulation 125, 996-1004 (2012).

20. Sirotin, N., Hoover, D. R., Shi, Q., Anastos, K. \& Weiser, S. D. Food insecurity with hunger is associated with obesity among HIV-infected and at risk women in Bronx, NY. PLoS One 9, e105957 (2014).

21. Mazidi, M., Kengne, A. P. \& Vatanparast, H. Food Security and Leukocyte Telomere Length in Adult Americans. Oxid. Med. Cell. Longev. 2017, 5427657 (2017).

22. Khayyatzadeh, S. S. et al. Nutrient patterns and their relationship to metabolic syndrome in Iranian adults. Eur. J. Clin. Invest. 46, 840-52 (2016).

23. Esmaillzadeh, A. et al. Fruit and vegetable intakes, C-reactive protein, and the metabolic syndrome. Am. J. Clin. Nutr. 84, 1489-97 (2006).

24. Esmaillzadeh, A. et al. Dietary patterns, insulin resistance, and prevalence of the metabolic syndrome in women. Am. J. Clin. Nutr. 85, 9108 (2007).

25. Esmaillzadeh, A. \& Azadbakht, L. Major dietary patterns in relation to general obesity and central adiposity among Iranian women. J. Nutr. 138, 358-63 (2008).

26. Esmaillzadeh, A. et al. Dietary patterns and markers of systemic inflammation among Iranian women. J. Nutr. 137, 992-8 (2007).

27. Mazidi, M. et al. Serum hs-CRP varies with dietary cholesterol, but not dietary fatty acid intake in individuals free of any history of cardiovascular disease. Eur. J. Clin. Nutr. 701454-1457 (2016).

28. Seligman, H. K., Bindman, A. B., Vittinghoff, E., Kanaya, A. M. \& Kushel, M. B. Food insecurity is associated with diabetes mellitus: results from the National Health Examination and Nutrition Examination Survey (NHANES) 1999-2002. J. Gen. Intern. Med. 22, 1018-23 (2007).

29. Seligman, H. K., Laraia, B. A. \& Kushel, M. B. Food insecurity is associated with chronic disease among low-income NHANES participants. J. Nutr. 140, 304-10 (2010).

30. Holben, D. H. \& Pheley, A. M. Diabetes risk and obesity in food-insecure households in rural Appalachian Ohio. Prev. Chronic. Dis. 3, A82 (2006).

31. Centers of Disease Control and Prevention. National Health and Nutrition Examination Survey: Plan and operations, 1999-2010. https:/www.cdc.gov/ nchs/data/series/sr_01/sr01_056.pdf. (2013).
32. Centers of Disease Control and Prevention. National Health and Nutrition Examination Survey: Anthropometry Procedures Manual. http://www.cdc.gov/ nchs/data/nhanes/nhanes_07_08/manual_an.pdf (2007).

33. Angelakis, E., Armougom, F., Million, M. \& Raoult, D. The relationship between gut microbiota and weight gain in humans. Future Microbiol. 7, 91-109 (2012).

34. National Health and Nutrition Examination Survey (NHANES). Laboratory Procedures Manual https://www.cdc.gov/nchs/data/nhanes/nhanes_11_12/201112_Laboratory_Procedures_Manual.pdf (2011).

35. Mohsen Mazidi, E. D. M. \& Maciej, Banach. The association of telomere length and serum 25-hydroxyvitamin D levels in US adults: the National Health and Nutrition Examination Survey. Arch. Med. Sci. 13, 61-5 (2017).

36. Konnov, M. V., Dobordzhinidze, L. M., Deev, A. D. \& Gratsianskii, N. A. Waist circumference below metabolic syndrome harmonizing criteria is associated with increased cardiovascular risk]. Kardiologiia 50, 23-7 (2010).

37. Ogbera, A. O. Prevalence and gender distribution of the metabolic syndrome Diabetol. Metab. Syndr. 2, 1 (2010)

38. Mazidi, M., Wong ND, Katsiki N, Mikhailidis DP, Banach M. Dietary patterns, plasma vitamins and Trans fatty acids are associated with peripheral artery disease. Lipids Health Dis. 16, 254 (2017)

39. National Center for Health Statistics. CFDCaPNHaNESA. http//www.cdc.gov/ nchs/nhanes.htm.

40. Mazidi, M., Gao, H. K. \& Kengne, A.P. Inflammatory Markers Are Positively Associated with Serum trans-Fatty Acids in an Adult American Population. J. Nutr. Metab. 2017, 3848201 (2017).

41. Mazidi, M., Gao, H.K., Shivappa, N., Wirth, M.D., Hebert, J.R. \& Kengne, A.P. The relationship of plasma Trans fatty acids with dietary inflammatory index among US adults. Lipids. Health. Dis. 16, 147 (2017).

42. Caudill, S. P., Schleicher, R. L. \& Pirkle, J. L. Multi-rule quality control for the agerelated eye disease study. Stat. Med. 27, 4094-106 (2008).

43. Risé, P., Eligini, S., Ghezzi, S., Colli, S.\& Galli, C. Fatty acid composition of plasma, blood cells and whole blood: relevance for the assessment of the fatty acid status in humans. Prostaglandins Leukot. Essent. Fatty Acids 76, 363-9 (2007).

44. Bickel, G. N. M., Price, C., Hamilton, W., Cook, J. Guide to measuring household food security. USDA, Food and Nutrition Service. http://www.fnsusdagov/FSEC/ FILES/FSGuide.pdf (2000).

45. National Center for Health Statistics. Analytic and reporting guidelines. http:// www.cdc.gov/nchs/data/nhanes/nhanes0304/ nhanesanalyticguidelinesdec2005.pdf (2015).

46. Leung, C. W., Epel, E. S., Ritchie, L. D., Crawford, P. B. \& Laraia, B. A. Food insecurity is inversely associated with diet quality of lower-income adults. J. Acad. Nutr. Diet. 114, 1943-53 (2014).

47. Becerra, M. B., Hassija, C. M., Becerra, B. J. Food insecurity is associated with unhealthy dietary practices among US veterans in California. Public Health Nutr. 20, 2569-2576 (2017).

48. Mello, J. A. et al. How is food insecurity associated with dietary behaviors? An analysis with low income, ethnically diverse participants in a nutrition intervention study. J. Am. Diet Assoc. 110, 1906-11 (2010).

49. Vuong, T. N., Gallegos, D. \& Ramsey, R. Household food insecurity, diet, and weight status in a disadvantaged district of Ho Chi Minh City, Vietnam: a crosssectional study. BMC Public Health 15, 232 (2015).

50. Tester, J. M., Laraia, B. A., Leung, C. W., Mietus-Snyder, M. L. Peer reviewed: Dyslipidemia and Food Security in Low-Income US Adolescents: National Health and Nutrition Examination Survey, 2003-2010. Prev. Chronic Dis. 13, (2016).

51. Widome, R., Neumark-Sztainer, D., Hannan, P. J., Haines, J. \& Story, M. Eating when there is not enough to eat: eating behaviors and perceptions of food among food-insecure youths. Am. J. Public Health 99, 822-8 (2009).

52. Johnson, R. K. et al. Dietary sugars intake and cardiovascular health. Circulation 120, 1011-20 (2009) 